\title{
Comparison between ab-initio and phenomenological modeling of the exchange couplings in diluted magnetic semiconductors: the
} case of $Z n_{1-x} C r_{x} T e{ }^{*}$

\author{
Georges Bouzerar ${ }^{1}$, Richard Bouzerar ${ }^{2}$, Josef Kudrnovský' ${ }^{3,4}$ and Timothy Ziman ${ }^{4}$ \\ ${ }^{1}$ Laboratoire Louis Néel 25 avenue des Martyrs BP 16638042 Grenoble Cedex 09 France. \\ ${ }^{2}$ Laboratoire de Physique de la Matière Condensée Université de Picardie Jules Verne, \\ 33 rue Saint Leu 80039 Amiens Cedex 01 France \\ 3 Institute of Physics Academy of Sciences, \\ Na Solvance 2 CZ-182 21 Prague 8 Czech Republic \\ ${ }^{4}$ Institut Laue Langevin BP 15638042 Grenoble France.
}

\begin{abstract}
Using a recently developed semi-analytical method (Self-Consistent Local RPA or SC-LRPA) we study the stability of the ferromagnetic phase in diluted magnetic systems where the exchange coupling between magnetic impurities are of RKKY form. A short discussion of the relevance of these calculations with respect to the ferromagnetism observed in diluted ferromagnetic materials is provided. Then, within a two step approach, we study ferromagnetism in $Z n_{1-x} C r_{x} T e$. In the first step of our study, we calculate the magnetic couplings between Mn impurities within the LDA. In the second step, we diagonalize the resulting effective Heisenberg Hamiltonian using the SC-LRPA. We also compare, when available, our calculations with Monte Carlo simulations and experimental measurements.
\end{abstract}

\footnotetext{
* To appear in "Proceedings of the International Conference MSM05, Agadir Maroc September 2005", Physica Status Solidi
} 
Diluted Magnetic Semiconductors materials have attracted considerable attention because of their technological potential in the domain of spintronics. These materials are particularly promising since a small concentration of magnetic impurities can already give rise to a relatively high Curie temperature [1, 2]. The key issue to obtain quantitative and reliable values of the Curie temperature is twofold. First, one has to be able to provide correct values for the exchange couplings between magnetic impurities. Secondly, one must treat properly the resulting effective Heisenberg Hamiltonian which describes the interaction between randomly distributed magnetic impurities on the lattice. By properly, we mean provide an accurate treatment of (i) thermal fluctuations and (ii) effects of disorder in the position of the impurities. Different approaches have been developed for the determination of the exchange integrals in III-V materials. There are essentially two different kind of methods. First, simple phenomenological approaches [3, 4] in which the band structure of the host material is described in a realistic manner by a Kohn-Luttinger Hamiltonian for the valence band states and a coupling $J_{p d}$ (or $V_{p d}$ ) between the itinerant carrier and the localized magnetic impurities. In these approaches, the coupling between localized magnetic impurity and the itinerant carrier is only treated perturbatively. However, within a simple single band model in which the local $J_{p d}$ coupling was treating non perturbatively within CPA, it has been shown that the perturbative treatment which leads to $J_{i j} \propto J_{p d}^{2}$ and thus to $T_{C} \propto J_{p d}^{2}$ is only restricted to very weak coupling $[6]$. A second class of approaches are based on first principle calculations. In these approaches, the coupling between $\mathrm{d}$ states of the magnetic impurities and host states is treated non-perturbatively, and this leads to realistic values of the exchange couplings. The main, and great, advantage of these approaches is that the couplings do not depend on adjustable parameters.

This manuscript is organized as follows. The first part is devoted to the study of the effects of thermal fluctuations and disorder on the stability of the ferromagnetic region as the density of itinerant carriers in diluted magnetic systems, with assumed RKKY-like exchange couplings. In view of our results, we discuss the relevance of such a phenomenological model to simulate the exchange couplings in actual diluted ferromagnetic semiconductors. In the second part, using realistic first principle calculations, we calculate the exchange couplings within the Tight-Binding LMTO approach in Cr doped ZnTe semiconductors. We then calculate the Curie temperature as a function of the magnetic impurity concentration for this material. 
Throughout this paper, we present calculations of the Curie temperature obtained using a recently developed Self-Consistent Local Random Phase Approximation (SC-LRPA) 7, 8]. The Curie temperatures calculated within SC-LRPA appear to be in very good agreement with Monte Carlo simulations where the same exchange couplings have been used [12]. However the SC-LRPA has several important advantages with respect to Monte Carlo calculations: it allows (i) to derive a direct semi-analytical expression for the Curie temperature (no extrapolation is required), (ii) the spins can be either quantum or classical, (iii) the calculations are very fast and (iv) correlation in the disorder can be included without any complication [9] . Within SC-LRPA the self-consistency is usually achieved after approximately 10-15 iterations, i.e. it requires the same number of diagonalizations of a $N_{i m p} \times N_{i m p}$ complex matrix. For comparison, for a given configuration of disorder, our calculations of Tc are at least 3 orders of magnitude faster than Monte Carlo calculations. Of course speed is not the only point; in the low temperature phase the SC-LRPA includes quantum effects that cannot be simulated classically. We now summarize the main steps of the SC-LRPA treatment. The disordered Heisenberg Hamiltonian which we consider is

$$
H_{e f f}=-\sum_{i j} J_{i j} \mathbf{S}_{i} \cdot \mathbf{S}_{j}
$$

The sum runs only over pairs of sites occupied by magnetic impurities. The spins are quantum with value $\mathrm{S}$. In the case of classical spins, we will properly perform the limit $S \rightarrow \infty$ at the end of the calculations. We now define the following retarded Green's function,

$$
G_{i j}(\omega)=\int_{-\infty}^{+\infty} G_{i j}(t) e^{i \omega t} d t
$$

where $G_{i j}(t)=-i \theta(t)\left\langle\left[S_{i}^{+}(t) ; S_{j}^{-}(0)\right]\right\rangle$.

After standard Tyablicov decoupling of the equation of motion of $G_{i j}(\omega)$ we obtain,

$$
\left(\omega-h_{i}^{e f f}\right) G_{i j}(\omega)=2\left\langle S_{i}^{z}\right\rangle \delta_{i j}-\left\langle S_{i}^{z}\right\rangle \sum_{l} J_{i l} G_{l j}(\omega)
$$

where the local effective field is,

$$
h_{i}^{e f f}=\sum_{l} J_{i l}\left\langle S_{l}^{z}\right\rangle
$$


For a given temperature and fixed disorder configuration, the local magnetization $\left\langle S_{i}^{z}\right\rangle$ has to be determined self-consistently at each impurity site. In order to close the set of equation we use Callen expression, which relate the local Green's function at site i to the local magnetization at this site [10].

$$
\left\langle S_{i}^{z}\right\rangle=\frac{\left(S-\Phi_{i}\right)\left(1+\Phi_{i}\right)^{2 S+1}+\left(S+1+\Phi_{i}\right) \Phi_{i}^{2 S+1}}{\left(1+\Phi_{i}\right)^{2 S+1}-\Phi_{i}^{2 S+1}}
$$

The local effective magnon occupation number reads,

$$
\Phi_{i}=\frac{-1}{2 \pi\left\langle S_{i}^{z}\right\rangle} \int_{-\infty}^{+\infty} \frac{I m G_{i i}(\omega)}{\exp (\omega / k T)-1} d \omega
$$

The previous set of equations allows us now to determine, at each temperature, the local magnetization at each impurity site, and the dynamical properties such as the dynamical structure factor.

To determine the value of the Curie temperature we take the limit $\left\langle S_{i}^{z}\right\rangle \rightarrow 0$ in the previous set of equations. We obtain,

$$
k_{B} T_{C}=\frac{1}{3} S(S+1) \frac{1}{N_{i m p}} \sum_{i} \frac{1}{F_{i}}
$$

where,

$$
F_{i}=\int_{-\infty}^{+\infty} \frac{A_{i i}(E)}{E} d E
$$

The local spectral function $A_{i i}(E)=-\frac{1}{2 \pi} \operatorname{Im}\left(\frac{G_{i i}(E)}{\lambda_{i}}\right)$. In the previous equation the factor $\lambda_{i}=\lim _{T \rightarrow T_{C}} \frac{\left\langle S_{i}^{z}\right\rangle}{m}$ ( $\mathrm{m}$ is the magnetization averaged over all impurity sites). It is interesting to note that the Curie temperature can be re-expressed in term of the eigenfunctions and eigenvalues of the following effective Hamiltonian, where the matrix elements are,

$$
\left(H_{e f f}\right)_{i j}=-\lambda_{i} J_{i j}+\delta_{i j} \sum_{l} \lambda_{l} J_{i l}
$$

This lead to,

$$
F_{i}=\sum_{\alpha} \frac{\left|\left\langle i \mid \Psi_{\alpha}\right\rangle\right|^{2}}{E_{\alpha}}
$$


Thus the Curie temperature becomes,

$$
T_{C}^{s c}=\frac{1}{3 N_{i m p}} S(S+1) \sum_{i}\left(\sum_{\alpha} \frac{\left|\left\langle i \mid \Psi_{\alpha}\right\rangle\right|^{2}}{E_{\alpha}}\right)^{-1}
$$

This expression implies that the nature (extended/localized) of the eigenstate will have important effects on the magnetic excitation spectrum and on the Curie temperature. A detailed discussion of these aspects will be provided elsewhere [11].

We now turn to the commonly used model of dilute magnetic systems where we place spins randomly, and independently, on a host lattice, and assume an interaction between each pair of spins of RKKY form with some density of carriers mediating the exchange. The carrier density is an important parameter, as it determines the scale of oscillation of the exchange. In Fig.1, we have plotted the Curie temperature for a fixed concentration of magnetic impurities as a function of the carrier density, normalized with respect to the concentration of impurities. We assume that the exchange couplings are of RKKY form and we including, the effects of disorder in an averaged way, i.e. by including an exponential damping of the couplings. Thus the interaction is taken to be

$$
J_{\mathrm{ij}}=J_{0} \exp \left(-\frac{r}{R_{0}}\right) \frac{\left(\sin \left(2 k_{F} r\right)-2 k_{F} r \cos \left(2 k_{F} r\right)\right)}{(r / a)^{4}}
$$

with Fermi vector $k_{F}$ determined by the density $k_{\mathrm{F}}=\left(3 \pi^{2} n_{c}\right)^{\frac{1}{3}}$ where $n_{c}=\gamma x$ is the density of carriers for impurity concentration $x$.

The curves of Curie temperature versus carrier concentration are shown for three different values of damping. Note that we do not consider in this work the effect of a short range super-exchange contribution; this is discussed in detail in Ref. [5]. For each case we show, for comparison (dotted lines), the Curie temperature estimated from mean-field Virtual Crystal Approximation (MF-VCA). This approximation has often been used, but, unlike ours, replaces the random lattice of impurities by a translationally invariant effective medium. We observe that as the carrier concentration increases from zero, our calculated Curie Temperatures have very different behaviour from the mean-field theory. Most dramatically, rather than increasing monotonically with carrier density, the Curie temperature saturates and vanishes at a density of carriers much smaller than the density of impurity moments. This is significant in that in doped $\mathrm{Ga}(\mathrm{Mn}) \mathrm{As}$, for example, it is clearly seen from simultaneous transport and magnetic measurements that the highest Curie temperatures are obtained 


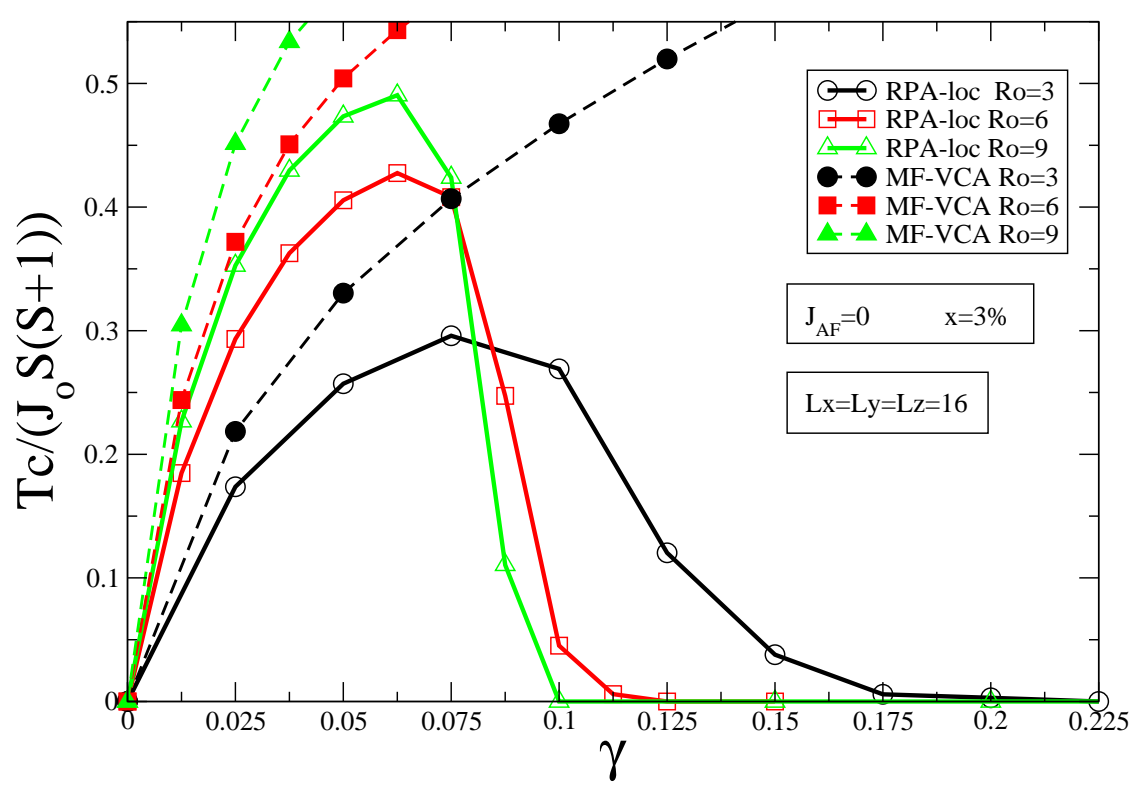

FIG. 1: Curie temperature as a function of the carrier density for a fixed concentration of magnetic impurities (3\%) and exchange couplings of RKKY form (see equation 12). The exponential cut-off in interactions is defined by the screening parameter $R_{0}$.

as the samples are annealed, giving carrier densities per moment approaching unity. Thus we conclude that simple RKKY interactions are not appropriate to describe the ferromagnetism in doped III-V semiconductors. The reasons for this had been obscured by the use of mean-field theories. The reason that the SC-LRPA shows the instability at higher carrier concentration is that includes the effect of the oscillating tail of the exchange constants, i.e. the frustration in the magnetic interactions. As damping becomes more short range, i.e. $R_{0}$ becomes smaller, the frustrating tail of interactions is reduced, as seen in Figure 1, but this is not enough to suppress the instability.

We learn from this that in order to explain observed ferromagnetism in diluted magnetic semiconductors we need to model the magnetic interactions more accurately. An approach that has proved successful, both in doped III-V [7] and II-VI materials, as we shall see below, is to use $a b$ initio methods to estimate the magnetic couplings at all distances including effects of the (disordered) band structure, correlations, screening treated within, for example Local Density Approximation (LDA). As the magnetic couplings can be calculated by a 


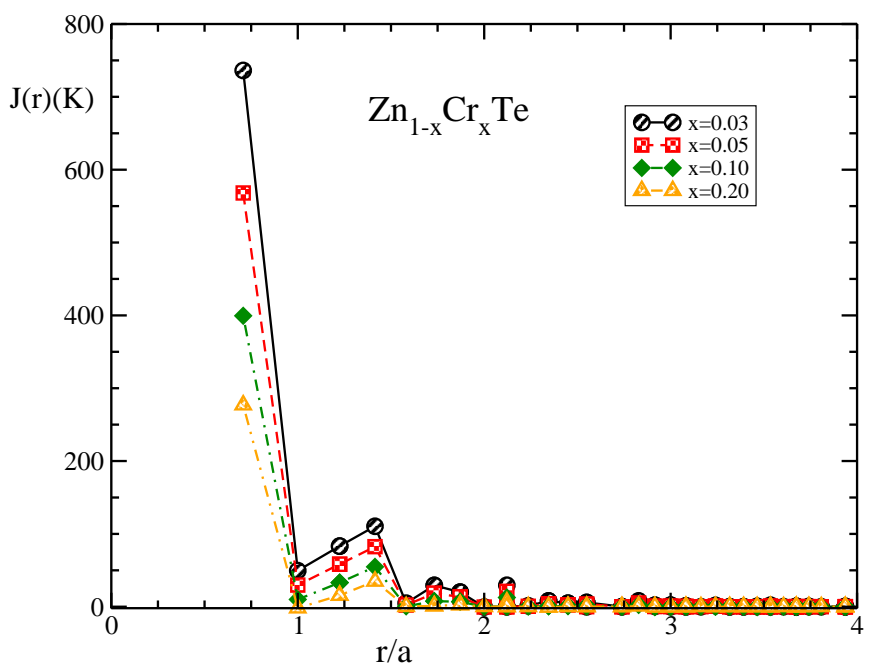

FIG. 2: Calculated Heisenberg exchange couplings (in Kelvin) as a function of the distance (in units of the lattice constant) between magnetic impurities for different concentration of $\mathrm{Cr} x=3 \%$ $, 5 \%, 10 \%$ and $20 \%$.

magnetic force theorem, i.e. in principle from the energies of the ground state perturbed by weak magnetic fields, they should be accurate. With the resultant Hamiltonian, which is specific to both the host material and the doping concentration (since disorder and doping effects are taken into account in the band structure), we will apply our method of SC-LRPA to estimate the Curie temperature. Within the class of II-VI semiconductors we turn now to the experimentally promising candidate for room temperature ZnTe doped with Cr.

First we show the exchange integrals between $\mathrm{Cr}$ moments deduced from our ab initio calculations. In Fig.2, we have plotted the magnetic exchange integrals between $\mathrm{Cr}$ impurities in ZnTe as a function of the distance, for different concentration. First, we clearly see that the frustration, which, we argued, could strongly suppress ferromagnetism in an RKKY model, is absent here. This is related to the fact that the Chromium dopants are close to the configuration $\mathrm{Cr}^{2+}$, i.e. do not, to a first approximation, introduce carriers into the II-VI host. In fact the band structure shows "half-metallicity": a finite density of states at the Fermi energy for the majority carriers and a gap for the minority carriers 14]. The couplings, while oscillating in magnitude, remain essentially ferromagnetic and exponentially damped. The ferromagnetic nature at short distances is attributed to the strong hybridization of the $\mathrm{Cr} 3 \mathrm{~d}$ levels with the Te 5 p states 14 . The damping has different origins, the effects of disorder (treated within CPA), the half-metallicity and the 
effects of the d levels. As the concentration of Cr increases, the couplings decrease at short distances keeping the same position of local minima.

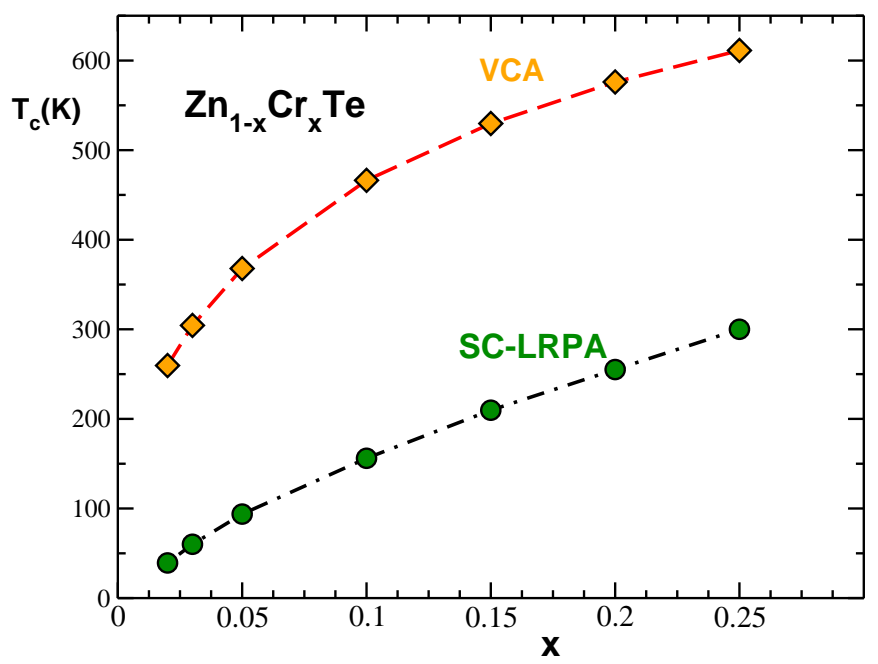

FIG. 3: Calculated Curie temperature within Self-Consistent Local RPA and Mean Field VCA for $Z n_{1-x} C r_{x} T e$ as a function of $x$.

In Fig. 3 we show the variation of the Curie temperature calculated within SC-LRPA as a function of the magnetic impurity concentration. A systematic average over at least 100 configurations of disorder has been taken for each concentration. For comparison, we also show the Mean-Field VCA results. While qualitatively the differences are not as dramatic as in Fig.1 in that both mean-field and our curve are monotonically increasing, quantitatively the predictions are very different. Most importantly, we believe our theory should be a reliable guide to the highest Curie temperatures that should be obtained for each concentration[7]., In particular we predict that room temperature should require concentrations around $25 \%$; the mean field VCA gives much lower values for this minimum concentration, about $2 \%$. Experimentally Saito et al [13] have found $\mathrm{T}_{C}=300 \pm 10$ for thin films with $x \approx 0.2$ which is close to our prediction $\left(\mathrm{T}_{C}(x=0.2)=265\right)$. Monte Carlo methods [12] for $\mathrm{Zn}(\mathrm{Cr}) \mathrm{Te}$ using the same exchange couplings were made for a few concentrations and are close to our curve. For example the Monte Carlo result is $\left(\mathrm{T}_{C}(x=0.2) \approx 300\right)$. We would conclude that the samples measured are close to the optimal Curie temperature for the concentration of uncorrelated impurities.

In conclusion, we have shown that the region of stability of ferromagnetism in diluted 
systems where the exchange couplings are RKKY-like is very narrow even when there is large damping. These couplings are very different from those obtained in ferromagnetic diluted semiconductors by means of first-principle calculations, and thus appear to be inappropriate to explain the wide region (in carrier concentration) of stability usually observed in these materials. We have calculated the variation of the Curie temperature in $Z n_{1-x} C r_{x} T e$ as a function of $\mathrm{Cr}$ density and found that room temperature can be achieved for densities above $x \approx 0.25$. This is important for this particular material and also illustrates how, by coupling first principle calculations of the exchange couplings with SC-LRPA, we may make useful predictions for a range of important new materials.

[1] H. Ohno, Science 281,951 (1998).

[2] K. W. Edmonds et al, Phys. Rev. Lett. 92,037201 (2004), K. W. Edmonds K.Y. Wang,R.P. Campion, B.L. Gallagher, C.T. Foxon, Appl. Phys. Lett. 81,4991 (2002). Additional values of $\mathrm{T}_{c}$ were provided by Edmonds et al ( private communication).

[3] T. Dietl, A. Haury and Y. Merle d'Aubigné, Phys. Rev. B 55, R3347 (1997)

[4] M. Abolfath, T. Jungwirth, J. Brum, and A. H. MacDonald, Phys. Rev. B 63, 054418 (2001)

[5] R. Bouzerar, G. Bouzerar and T. Ziman submitted to Phys Rev B.

[6] G. Bouzerar, J. Kurdnovskỳ and P. Bruno, Phys. Rev. B 68, 205311 (2003)

[7] G. Bouzerar, T. Ziman and J. Kudrnovskỳ, Eur. Phys. Letters, 69, 812-818 (2005).

[8] G. Bouzerar, T. Ziman and J. Kudrnovskỳ, Phys. Rev. B 72, 125207 (2005).

[9] G. Bouzerar, T. Ziman and J. Kudrnovskỳ, Appl. Phys. Lett. 854941 (2004).

[10] H. B. Callen, Phys. Rev. 130,890 (1963).

[11] G. Bouzerar et al., in preparation.

[12] L. Bergqvist, O. Eriksson, J. Kudrnovský, P.A. Korzhavyi, and I. Turek, Phys. Rev. Lett. 93, $137202(2004)$.

[13] H. Saito,V. Zayets, S. Yamagata and K. Ando , Phys. Rev. Letters 90, 207202 (2003)

[14] Q. Wang, Q. Sun,P. Jena and Y. Kawazoe, J. Appl.Phys. 97043904 (2005). 